\title{
Analysis of Doppler frequency shift response mechanism based on OFDM system
}

\author{
$\mathrm{Li} \mathrm{Ke}^{1}$, Sun Zhiguo ${ }^{2}$, Ning Xiaoyan ${ }^{3}$ and Diao Ming ${ }^{4}$ \\ ${ }^{1}$ Harbin Eingineering University,Harbin, China.
}

\begin{abstract}
Absrtact: OFDM system is widely used in mobile communication because of its high spectral efficiency and strong anti-multipath capability. However, in high dynamic scenarios, the Doppler shift between the transmitter and the receiver destroys the orthogonality between the sub-carriers, resulting the interference between the sub-carriers and distortion of the signal, which greatly degrades the system performance. In this paper, a mathematical model of Offset-OFDM system was established and the Doppler response formula of OFDM system in high white channel was derived. Furthermore, we investigated the factors that influence the performance of OFDM. This study laid the foundation for determining OFDM system parameters in high dynamic scenarios.
\end{abstract}

\section{INTRODUCTION}

As one of the key technologies of $4 \mathrm{G}$ communication, OFDM technology has strong anti-multipath capability. Each sub-carrier in the system is strictly orthogonal, thus, they don't interfere with each other and this system has a good spectrum utilization. Therefore, the system has attracted great attention in the high-speed wireless communication environment with limited resources and has been adopted as the standard of DAB / DVB system. However, with the rapid development of communication technology, it is a great challenge to design OFDM systems in high dynamic $s^{1}$ cenarios. Due to the Doppler shift in high dynamic scenarios, each sub-carrier is no longer orthogonal, resulting in inter-carrier interference, greatly reducing the performance of communication systems. In recent years, a large number researches have studied the influence of Doppler shift on OFDM. In the works of Zhao and Haggma, Pollet et al.,carrier interference ratio (CIR) was proposed as an evaluation of inter-carrier interference. Reference [1-3] investigated the relationship between CIR and BER, and concluded that the higher the CIR, the lower the BER. In Reference [4], using modulation signal of BPSK and QPSK as an example, the formula of BER with normalized frequency offset in high white channel and Rayleigh channel of OFDM system was derived. But they have not studied the influence of subcarrier number and modulation mode on OFDM system.

In this paper, the mathematical relationship between received signal and normalized frequency offset in high white channel was established in OFDM system. The variation law of inter-carrier interference was analyzed.
In addition, the influence of two important parameters (modulation mode and subcarrier number) on OFDM system performance was investigated.

\section{DOPPLER SHIFT RESPONSE MODEL FOR OFDM SYSTEM}

\subsection{Basic principles of OFDM system}

The OFDM transceiver-receiver block diagram is shown in Figure 1: Input binary streams are first converted through series-parallel and constellations mapped into sequences: $X(k), k=0,1 \ldots N-1, N$ is the number of sub carriers..IFFT is used for carrier modulation, and the sequence is modulated to each orthogonal sub-carrier to ensure that the sub-carriers are orthogonal, then the modulated signal can be expressed as:

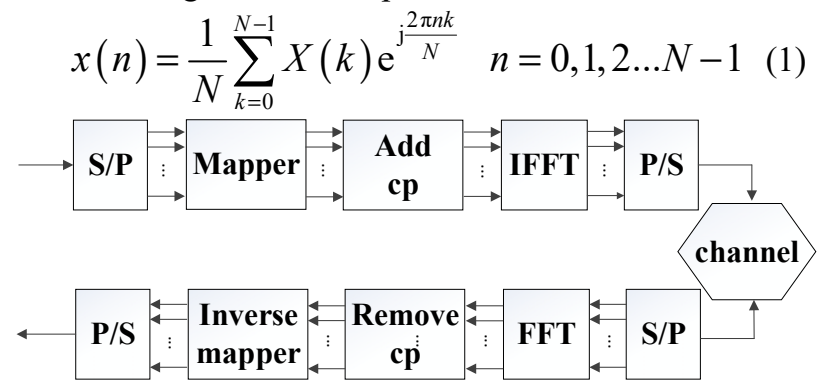

Figure 1.OFDM system block diagram

In order to eliminate ISI to the greatest extent, it is necessary to insert protection interval between OFDM symbols, and to ensure that the protection interval length is larger than the maximum delay expansion of wireless channel, so ISI can be eliminated. Adding cyclic prefix means copying the last several sample points of each OFDM symbol in front of the OFDM symbol, so as to

\footnotetext{
${ }^{\text {a }}$ Corresponding author: Ning xiaoyan :ningxiaoyan $@$ hrbeu.edu.cn.
} 
ensure that the number of sub-carriers in the integration period of FFT is still an integer, thus ensuring the orthogonality between sub-carriers. Because this paper mainly discussed the effect of Doppler shift on OFDM, we can ignore the cyclic prefix. After the signal passes through the channel, the cyclic prefix is removed at the receiver. FFT operation and inverse mapping can recover the original signal.

\subsection{Doppler shift response model}

Because the Doppler frequency shift exists at both ends of the transceiver, the signal received at the receiving end can be expressed as a formula (2). (The influence of Gauss white noise is neglected in the derivation and analysis.)

$$
Y(n)=\frac{1}{N} \sum_{k=0}^{N-1} X(k) \mathrm{e}^{\mathrm{j} \frac{2 \pi n(k+\varepsilon)}{N}}
$$

In the formula(2), $n=0,1 \ldots N-1, \varepsilon$ is normalized frequency offset, defined as frequency offset divided by sub carrier interval. That is $\varepsilon=\frac{\Delta f}{B}$ 。 After FFT calculation, the sequence of receiving ends is

$$
y(m)=\sum_{n=0}^{N-1} Y(n) \mathrm{e}^{\mathrm{j} \frac{2 \pi n m}{N}}
$$

After introducing the formula (2) into (3), it is simplified:

$$
\begin{aligned}
& y(m)=\frac{1}{N} \sum_{n=0}^{N-1} \sum_{k=0}^{N-1} x(k) \mathrm{e}^{\mathrm{j} \frac{2 \pi n}{N}(k+\mathcal{E}-m)} \\
& =\frac{1}{N} \sum_{n=0}^{N-1} x(m) \mathrm{e}^{\mathrm{j} \frac{2 \pi n}{N} \varepsilon}+\frac{1}{N} \sum_{n=0}^{N-1} \sum_{\substack{k=0 \\
k \neq m}}^{N-1} x(k) \mathrm{e}^{\mathrm{j} \frac{2 \pi n}{N}(k+\mathcal{E}-m)}
\end{aligned}
$$

According to the formula:

$$
\sum_{n=0}^{k-1} \exp (u)=\frac{1-\mathrm{e}^{k}}{1-e} \quad \text {,Formula (3) can be simplified: }
$$

$$
\begin{aligned}
& y(m)=\frac{1}{N} x(m) \frac{1-\mathrm{e}^{2 \pi \varepsilon}}{1-\mathrm{e}^{\frac{2 \pi \varepsilon}{N}}}+\frac{1}{N} \sum_{\substack{k=0 \\
k \neq m}}^{N-1} x(k) \frac{1-\mathrm{e}^{\mathrm{j} 2 \pi(k+\varepsilon-m)}}{1-\mathrm{e}^{\mathrm{j} \frac{2 \pi}{N}(k+\varepsilon-m)}} \\
& =\frac{1}{N} x(m) \mathrm{e}^{\mathrm{j} \pi\left(1-\frac{1}{N}\right) \varepsilon} \frac{\sin \pi \varepsilon}{\sin \frac{\pi \varepsilon}{N}}+\frac{1}{N} \sum_{\substack{k=0 \\
k \neq m}}^{N-1} x(k) \frac{1-\mathrm{e}^{\mathrm{j} 2 \pi(k+\varepsilon-m)}}{1-\mathrm{e}^{\mathrm{j} \frac{2 \pi}{N}(k+\varepsilon-m)}} \\
& =x(m) \mathrm{e}^{\mathrm{j} \pi\left(1-\frac{1}{N}\right) \varepsilon} \frac{\sin \pi \varepsilon}{\mathrm{N} \sin \frac{\pi \varepsilon}{\mathrm{N}}}+\sum_{\substack{k=0 \\
k \neq m}}^{N-1} x(k) \mathrm{e}^{\mathrm{j}(k+\varepsilon-m) \pi\left(1-\frac{1}{N}\right)} \\
& \times \frac{\sin \pi(k+\varepsilon-m)}{N \sin \left(\frac{\pi(k+\varepsilon-m)}{N}\right)} \\
& =x(m) S(m, m)+\sum_{\substack{k=0 \\
k \neq m}}^{N-1} x(k) S(k, m)
\end{aligned}
$$

$S(k, m)$ is the subcarrier interference coefficient: It can be seen that when the noise is ignored, the received signal is composed of the amplitude and phase changed target signal and the interference between the other sub-carriers, which causes the receiver signal to exceed the decision interval and cause error code.

Frequency offset can be divided into integer frequency offset and fractional frequency offset.

Formula (5) shows that when $\mathcal{E}$ is integer, $S(k, m)$ is zero, and there is no inter-carrier interference. At this time, $Y(m)=x(m-\varepsilon)$. When it is a decimal, the received signal is subject to three kinds of interference: the phase rotation and amplitude attenuation of the signal, the interference between subcarriers, and the interference of white Gaussian noise.

\section{DOPPLER FREQUENCY SHIFT RESPONSE MECHANISM AND SIMULATION ANALYSIS OF OFDM SYSTEM}

\subsection{Intercarrier interference analysis}

Figure 2 shows the curve of $S(k, m)$ when $\mathcal{E}$ is 0.05 , $0.2,0.5$. It can be seen that when the $\mathcal{E}$ is relatively small, the received target subcarrier is mainly interfered by adjacent subcarriers. The larger the $\mathrm{X}$, the greater the interference of the target subcarriers from the far sub carriers. And $\sum_{k=0}^{N-1}|S(k)|^{2}=1$, document [5] gives the proof of this formula.

Carrier Interference Ratio (CIR) is an important index to measure ICI. It is defined as the ratio of expected signal power to inter-carrier interference power. Assuming that the transmitted signal is zero mean and identically distributed,then:

$C I R=\frac{\mathrm{E}\left[|X(m) S(m . m)|^{2}\right]}{\mathrm{E}\left[\left|\sum_{\substack{k=0 \\ k \neq m}}^{N-1} X(m) S(k . m)\right|^{2}\right]}=\frac{|S(0)|^{2}}{\sum_{\substack{k=0 \\ k \neq m}}^{N-1}|S(k . m)|^{2}}$ 


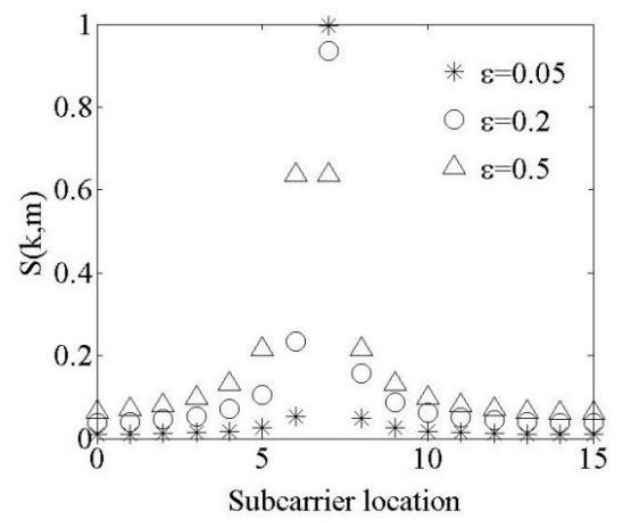

Figure 2 Inter carrier interference simulation

Figure 3 shows the curve along with CIR. It can be seen that with the increase of $\mathcal{E}$, CIR will decrease.

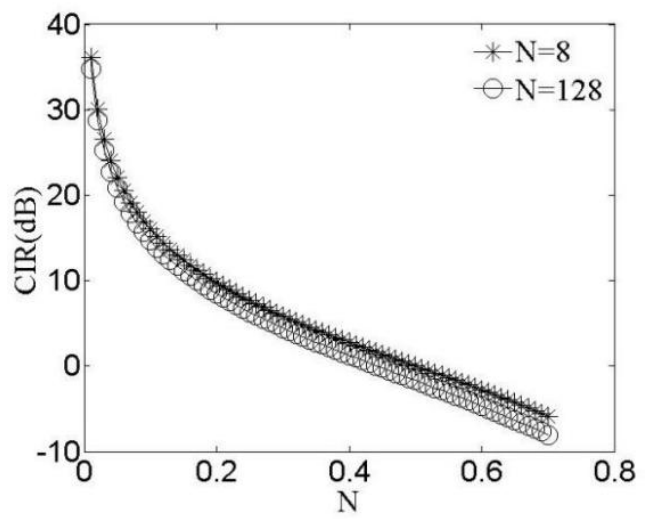

Figure 3.the simulation diagram of CIR

\subsection{Influence of subcarrier number on CIR}

In the previous section, we see that CIR is a function of $\mathrm{N}$, which is the subcarrier number. Because of the large number of subcarriers in the general OFDM system, we only discuss the case when $\mathrm{N}$ is large. Then:

$$
C I R=\frac{|S(0)|^{2}}{\sum_{\substack{k=0 \\ k \neq m}}^{N-1}|S(k . m)|^{2}}=\frac{|S(0)|^{2}}{1-|S(0)|^{2}}=\frac{1}{\frac{N^{2} \sin ^{2} \frac{\pi \varepsilon}{N}}{\sin ^{2} \pi \varepsilon}-1}
$$

when $\mathrm{N}$ is large:

$$
C I R \approx \frac{1}{\frac{\pi^{2} \varepsilon^{2}}{\sin ^{2} \pi \varepsilon}-1}
$$

According to formula (8), when $N$ is relatively large, CIR is not affected by $N$. Fig. 4 shows the variation curve of CIR with $N(\mathcal{E}=0.2)$. It can be seen that when $\mathrm{N}$ is greater than 10 , the CIR is basically unchanged. Because the number of subcarriers of general OFDM systems is in hundreds or thousands, the influence of $N$ on OFDM systems can be neglected when designing OFDM systems with only Doppler shift.

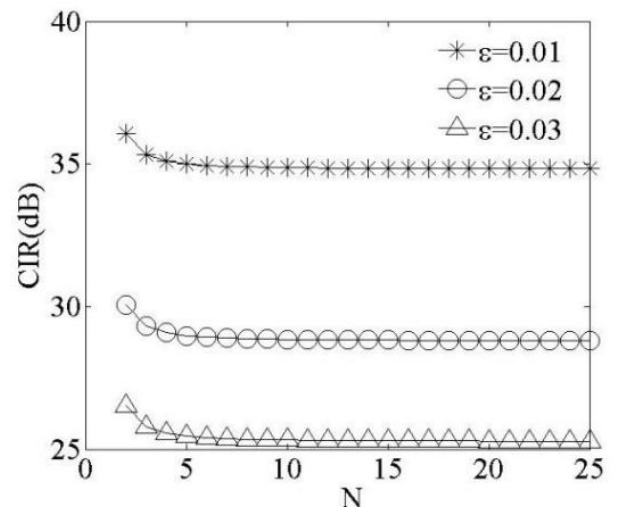

Figure 4.Curve of CIR with subcarrier number

\subsection{Influence of modulation mode on OFDM system performance}

In the case of ignoring the noise, the factors that affect the decision error of the received signal mainly consist of the attenuation of the signal itself and the inter-carrier interference. According to the previous section, modulation mode does not affect inter carrier interference. However, due to the different modulation modes, the decision intervals of constellations are different. When the signal itself has phase rotation and amplitude attenuation, the influence on the signal decision is also different. The most common modulation modes in OFDM system, 16PSK and 16QAM, were taken as examples to compare and analyze the influence of signal attenuation on decision.

The 16PSK constellation diagram and decision interval are shown in Figure 5. When the received signal exceeds the decision interval of the shadow part, a decision error occurs. Because the decision interval is sector and the amplitude variation does not affect it, the system will make a decision error when the noise is neglected and the following relation is satisfied (not losing generality, assuming the frequency offset is positive):

$$
\varepsilon \geq \frac{1}{16}
$$

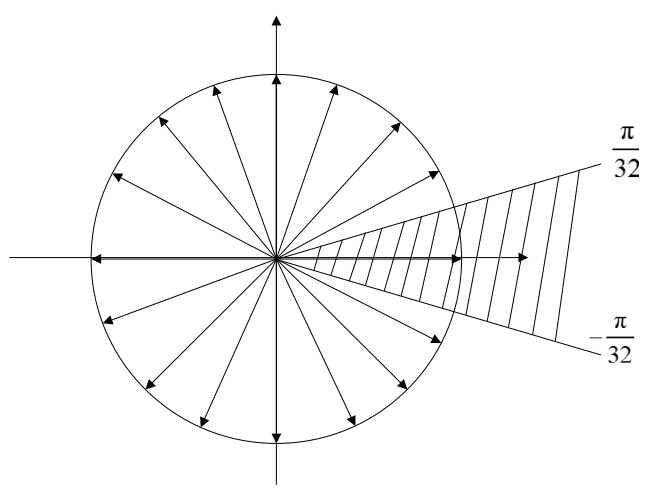

Figure 5.16PSK constellation and decision region

The constellation diagram and decision region of 16QAM are shown in Fig. 6. Because the constellation diagram of 16QAM is square, both amplitude and phase change will affect the correctness of the decision. The minimum distance is assumed to be $\mathrm{d}$. It is only 
necessary to determine whether the signal is still in the judgment region when its constellation phase rotates $\pi \varepsilon$ and the amplitude decays $\frac{\sin (\pi \varepsilon)}{\pi \varepsilon}$ under the condition of $\varepsilon=\frac{1}{16}$.

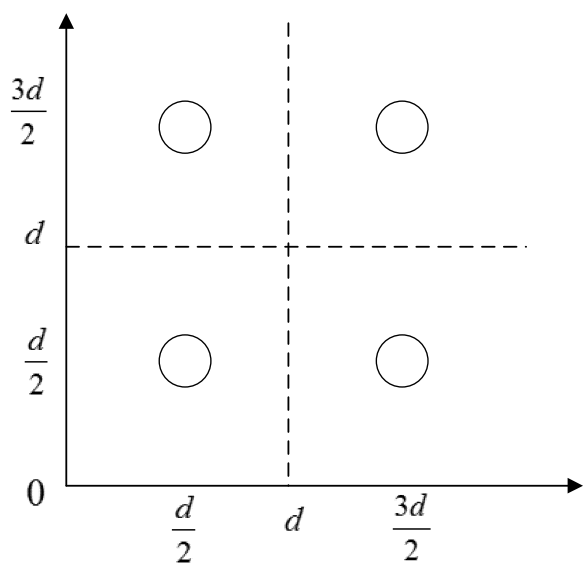

Figure 6.16QAM constellation and decision region

Take the coordinate point $\left(\frac{3 d}{2}, \frac{d}{2}\right)$ as an example: after attenuation, the horizontal and vertical coordinates of the signal are $(X, Y)$.

$$
\begin{aligned}
& X=\sqrt{\left(\frac{3}{2} d\right)^{2}+\left(\frac{d}{2}\right)^{2}} \frac{\sin \left(\frac{\pi}{16}\right)}{\frac{\pi}{16}} \cos \left(\arctan \left(\frac{1}{3}\right)+\frac{\pi}{16}\right) \\
& =1.36 d>d \\
& Y=\sqrt{\left(\frac{3}{2} d\right)^{2}+\left(\frac{d}{2}\right)^{2}} \frac{\sin \left(\frac{\pi}{16}\right)}{\frac{\pi}{16}} \sin \left(\arctan \left(\frac{1}{3}\right)+\frac{\pi}{16}\right) \\
& =0.78 d<d
\end{aligned}
$$

It is known that the signal after attenuation is still in the judgment area that it belongs to. Similarly, the other four points are also decayed after their attenuation. It can be concluded that 16QAM has stronger resistance to Doppler shift than 16PSK modulation in the case of high signal-to-noise ratio. Figure 7 shows the performance comparison curves of the OFDM system after 16QAM and 16PSK modulation respectively. The setting of signal to noise ratio $(30 \mathrm{~dB})$ is to reduce the influence of noise. The sub carrier $\mathrm{N}$ is set to 128 . It can be seen from the diagram. The ability of 16QAM to resist Doppler shift is stronger than that of $16 \mathrm{PSK}$.

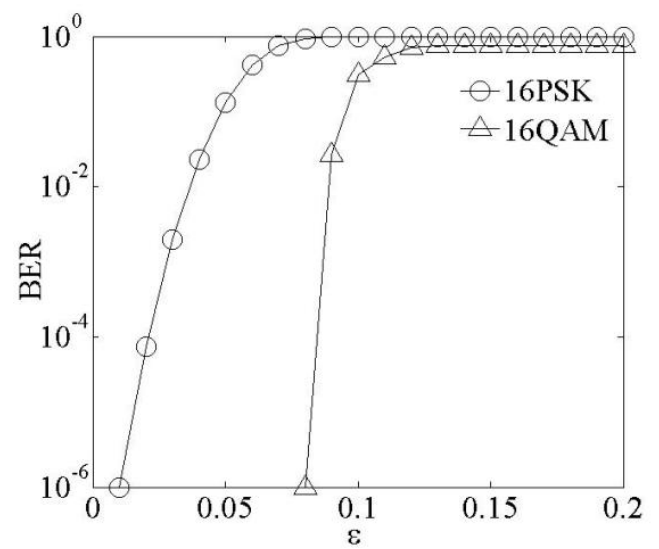

Figure 7.Performance simulation diagram of OFDM system with 16PSK and 16QAM modulation respectively under the same frequency offset and SNR

\section{SUMMARY}

In this paper, the mathematical model of Doppler frequency shift on OFDM system was established, the characteristics of inter-carrier interference (ICI) were studied, and the influence of subcarrier number and carrier modulation on the performance of OFDM system were investigated. General conclusions are drawn.

\section{References}

1. Y.Zhao and S.Haggman,"Sensitivity to Doppler shift and carrier frequency errors in OFDM systems-The consequences and solutions,"in IEEE 46th Vehicular Technology Conf.,vol.3,Apr.1996,pp.1564-1568.

2. T.Pollet,M.V.Bladel and M.Moeneclaey,'BER sensitivity of OFDM systems to carrier frequency offset and Wienerphases noise,"IEEE Trans.Commun,vol.43,pp.191-193.Feb.-Apr.1995.

3. K.Sathananthan and C.Tellambura,"Probability of Error Calculation of OFDM Systems With Frequency Offset,"IEEE Transactions on communications, VOL.49,NO.11,NOVEMBER 2001.

4. J.Ahn and H.S.Lee,"Frequency domian equalization of OFDM signal over frequency nonselective Raleigh fading.channels,"Electron.Lett.vol.29,no.16,pp.147 6-1477,Aug.1993.

5. H.Moose,"A technique for orthogonal frequency division multiplexing frequency offset correction,"IEEE

Trans.Commun,vol.42,pp.2908-2914,Oct.1994. 